\title{
Wind-terrain effects on firebrand dynamics
}

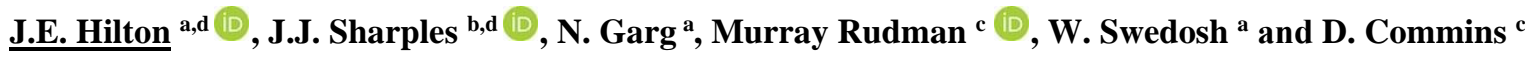 \\ ${ }^{a}$ Data61, CSIRO, Clayton South, VIC 3169, Australia \\ ${ }^{b}$ School of Science, UNSW, Canberra, ACT 2600, Australia \\ ${ }^{c}$ Department of Mechanical \& Aerospace Engineering, Monash University, VIC 3800, Australia \\ ${ }^{d}$ Bushfire and Natural Hazards Cooperative Research Centre, East Melbourne VIC 3002, Australia \\ Email:james.hilton@csiro.au
}

\begin{abstract}
Despite its importance in bushfire propagation, firebrand transport and the spotting process are still poorly understood, and there is no definitive model that can adequately emulate the spotting process in general. The dynamics of firebrands are difficult to predict due to the complex flow structure resulting from the interaction of a buoyant plume with a boundary layer wind field. Understanding the nature of this flow structure, especially for complex terrain, is essential for determining the likely path of firebrands and subsequent distributions of new spot fires and risk levels on structures downwind from the fire.
\end{abstract}

Although several prior computational modelling studies have carried out investigations of firebrand transport, the effect of the terrain has not previously been taken into account. It is well known that topography can significantly affect ember generation. For example, the enhanced intensity of a fire running up a steep slope can generate a large number of embers. More generally, terrain-modified flows and the strong turbulence associated with leeward slopes and flow around other prominent topographic features may have a pronounced effect on the transport of firebrands. Moreover, modes of dynamic fire propagation such as vorticity-driven lateral spread and eruptive fire spread in canyons involve a coupling between the fire, the terrain and the prevailing winds and so can affect the rate at which firebrands are produced as well as their subsequent transport.

In this study we use a coupled computational fluid dynamic (CFD) and Lagrangian particle approach to model the transport of firebrands. The model is applied to two different terrain scenarios to investigate the flow dynamics, firebrand trajectories and landing patterns resulting from the interaction with the terrain. The first scenario is a line of fire on the lee slope of a ridge burning perpendicular to an incident wind flow. The second scenario is a fire burning in a canyon aligned with the wind. The simulations indicate that the addition of terrain adds a further level of complexity to the flows generated by interaction between the wind and the fire. The terrain appears to modify the counter-rotating vortex pair in the plume structure. For the fire in the lee of the ridge line, the wind-terrain interaction resulted in a flattening and tilting of the counter-rotating vortex pair and enhanced regions of recirculation at the edges of the fire, which were conducive to lateral transport of embers. For the fire in the canyon, the channelling of the winds up the canyon resulted in the formation of a single jet-like vortex transporting firebrands upwards and over the top of the canyon. We hypothesise that this effect is caused by the shape and alignment of the canyon, which forces the vortex pair to merge into a single vortex.

Keywords: $\quad$ Wildfire modelling, Firebrand, Computational Fluid Dynamics, Spark 


\section{INTRODUCTION}

Firebrands are small pieces of flaming vegetation transported downwind of a wildfire. These have the potential to start secondary fires away from the fire front and the mass transport of firebrands and subsequent ignition of new fires represent a major and unpredictable mode of wildfire propagation (Koo et al. 2010). Typically, firebrands fall into three classes: short, medium and long-range (Cruz et al., 2012). Short-range firebrands are not lofted by the fire and their motion is dominated by the ground-level winds driving the fire. These are found closest to the fire, typically at tens to hundreds of metres from the front. Ember storms, which are the greatest cause of house loss at the wildland-urban interface (Blanchi, 2006), fall into this category. Medium range firebrands are lofted by the buoyant plume of the fire and fall vertically from the plume downwind of the fire in ember showers (typically at a distance hundreds of meters to a few kilometres). Long range firebrands are large pieces of flaming material, such as Australian stringybark, which have long burnout times and aerodynamic characteristics that allow long lofting times and fall at distances many kilometres away from the active fire (Ellis, 2013). The dynamics of medium and long-range firebrands are very difficult to predict as they are transported in turbulent flow structures. However, prediction of firebrand transport is essential for early warnings in the event of a wildfire and for assessing risk from hypothetical fire scenarios.

While modelling firebrand transport in turbulent buoyant plumes has been covered in several studies the role of terrain in firebrand transport has, so far, received less attention. Koo et al. (2012) carried out an in-depth study of firebrand transport, taking into account factors such as the shape of the particle and modelling the dynamics of the system using a coupled CFD and combustion model. They presented detailed dynamics of the combustion, flow structure and distribution of firebrands landing on the ground. Thurston et al. (2017) investigated the role of plume structure in long-range firebrand dispersal and carried out numerical simulations of firebrand transport over domains of several kilometres. They showed that turbulence and wind speed had a significant effect in the distribution of the firebrands and noted the role of the well-known plume counterrotating vortex pair in the transport dynamics. Wadhwani et al. (2017) modelled the trajectory of firebrands using the NIST Fire Dynamics Simulator (FDS) to compare with the experimental results. More recently Wadhwani et al. (2019) have also modelled landing distributions for embers at the edge of a forest, providing detailed information of the flow structure and transition of the wind field. Thomas et al. (2019) used a numerical weather prediction model to examine the validity of the terminal-velocity assumption in long-range ember transport.

These previous studies have, however, been carried out on flat terrain, thus limiting their applicability in real world. Terrain strongly affects the flow of wind and, in turn, the dynamics of firebrands in a wind stream. Here we investigate two fire scenarios that occur in connection with terrain features commonly involved in dangerous wildfire development. The first is a fire line burning in the lee of a ridge under a strong perpendicular wind. Such a scenario could arise as fires spreading along a ridgeline or by vorticity-driven lateral spread (Sharples et al. 2012, Simpson et al. 2014, Raposo et al. 2015). Vorticity-driven lateral spread, in particular, can lead to unintuitive fire behaviour with implications for firefighting strategies. Such fire behaviour also likely leads to enhanced spot fire formation due to an elongated front proximal to strong winds at a ridge, and enhanced ember generation due to the effects of strong vorticity. The second scenario involves a canyon with a strong incident wind aligned along its axis. Such configurations have been implicated in connection with eruptive fire development (Viegas and Pita 2004, Sharples et al. 2010), and have been highlighted in the context of wildfire propagation and firefighter safety (Lahaye et al. 2018). However, modelling of firebrand transport and subsequent spot fire distributions has not been previously been carried out on such terrain.

\section{METHODLOGY}

In this study we limit the dynamics of the system to the interaction of a three-dimensional wind field with terrain and the subsequent transport of particles representing firebrands in the flow fields. To simplify the modelling, the combustion and spread of the fire is not modelled and, instead, the fire line is held fixed and acts as a volumetric heat source.

The equations of motion for the air flow are the Navier-Stokes equations plus a thermal advection term. The system is assumed incompressible, $\nabla \cdot \mathbf{u}=0$, where $\mathbf{u}$ is the air velocity vector $\left(\mathrm{m} \mathrm{s}^{-1}\right)$. The Boussinesq approximation is used to couple the thermal term with the momentum equation. The momentum equation is given by:

$$
\frac{\partial \mathbf{u}}{\partial t}=-\mathbf{u} \cdot \nabla \mathbf{u}+\eta \nabla^{2} \mathbf{u}-\alpha T \boldsymbol{g}
$$


where $\eta$ is an effective turbulent viscosity, $\alpha$ is the thermal expansion coefficient of air, $T(K)$ is the air temperature above ambient temperature and $\boldsymbol{g}=(0,0,-9.8)$ is the gravitational acceleration vector. Molecular viscosity is ignored here as turbulent viscosity dominates over this term. The thermal equation is given by:

$$
\frac{\partial T}{\partial t}=-\mathbf{u} \cdot \nabla T+\kappa \nabla^{2} T+q
$$

where $q$ is the thermal source term $\left(K s^{-1}\right)$ and $\kappa$ is the turbulent diffusion coefficient. The thermal diffusivity of air is assumed to be negligible as the dominant contribution to the diffusion term is turbulent diffusion.

The force on a firebrand is governed by the drag and gravitational force:

$$
m \frac{\partial \mathbf{v}}{\partial t}=\frac{1}{2} \rho c_{D} A|\mathbf{u}-\mathbf{v}|(\mathbf{u}-\mathbf{v})+\boldsymbol{g}
$$

where $m$ is the mass of the firebrand, $\mathbf{v}$ is the firebrand velocity vector, $\rho$ is the air density $\left(\mathrm{kg} \mathrm{m}^{-3}\right), c_{D}$ the coefficient of drag for the firebrand and $A\left(\mathrm{~m}^{2}\right)$ is the cross-sectional area of the firebrand perpendicular to the flow.

\subsection{Numerical modelling}

The equations of motion for the air are modelled using a finite difference pressure-projection method (Chorin, 1967). The domain was discretised into cubical cells for the air flow, whereas the firebrands were modelled as spherical Lagrangian particles. A schematic diagram of the set-up for a fire source along a ridge line is shown in Fig. 1. A synthetic two-dimensional digital elevation model of the height of the land surface was used to define the terrain. The simulations in this study used a grid cell size $\Delta$ of $10 \mathrm{~m}$.

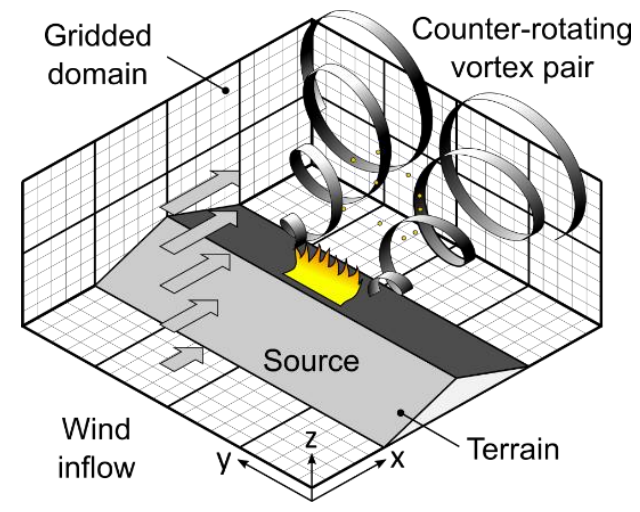

Figure 1. Schematic illustration of the computational set up and domain.

Turbulence was modelled using the Wall-Adapting Large Eddy Simulation (WALE) method (Nicoud et al., 1999). Unlike other LES methods, the WALE method is designed to dynamically adapt to the closest surface ('wall') rather than requiring a prescribed distance as an input to the model. The method has been shown to match DNS simulations for a range of flow types (Nicoud et al., 1999). Details of the computational implementation of the WALE method are given in Hilton et al. (2012). The computation of Eqs. (1-3) were carried out using GPU-accelerated implementations available within the Spark framework (Spark, 2019). The advection terms in Eqs. $(1,2)$ were computed using $3^{\text {rd }}$ order weighted essentially non-oscillatory (WENO) methods (Liu et al., 1994) and the diffusion terms were modeled using a $2^{\text {nd }}$ order finite difference stencil. The Laplacian resulting from the pressure projection update was calculated using a multigrid scheme (Wesseling, 1992).

Firebrands were produced within the flaming region with an average production rate of 1 firebrand every 10 seconds per cell. The equations of motion for the firebrands, Eq. (3), were calculated using an adaptive $3^{\text {rd }}$ order Bogacki-Shampine Runge-Kutta method. An adaptive high-order method was found to be necessary due to the large relative accelerations experienced by small firebrands in the plume. Due to the density difference between air and the firebrand, buoyancy on the particle was assumed negligible, as were any other flowincluded forces such as shear (Saffman) or rotational (Magnus) lift forces. The air speed at the position of each firebrand, needed for $\mathbf{u}$ in Eq. (3) was interpolated tri-linearly from the flow grid. All firebrands were modelled as spheres of radius $2.5 \mathrm{~mm}$ and density $250 \mathrm{~kg} \mathrm{~m}^{-3}$. 
The boundary conditions on the domain were a no-flow zero velocity condition on the base and any cells below the land surface, free flow conditions on the downwind, side and upper boundaries. The inflow boundary used a logarithmic wind profile of the form:

$$
u_{x}=w_{10} \ln \left(\frac{z}{z_{0}}\right) \ln \left(\frac{10}{z_{0}}\right)^{-1}
$$

where $w_{10}$ is the $10 \mathrm{~m}$ wind speed $\left(\mathrm{m} \mathrm{s}^{-1}\right), z$ is the vertical height $(\mathrm{m})$ and $z_{0}$ is the roughness length $(\mathrm{m})$, taken to be $z_{0}=0.25 \mathrm{~m}$ for the cases in this study.

The fire was set as a fixed heat source for all simulations. The thermal source term can be estimated by:

$$
q=\frac{Q}{\rho c_{p}}=\frac{H}{\rho c_{p}} \frac{\partial w}{\partial t}
$$

where $Q$ is the volumetric heat release rate $\left(\mathrm{J} \mathrm{m}^{-3} \mathrm{~s}^{-1}\right), c_{p}$ is the specific heat capacity of air $\left(\mathrm{J} \mathrm{kg}^{-1} \mathrm{~K}^{-1}\right), H$ is the heat of combustion of the fuel $\left(\mathrm{J} \mathrm{kg}^{-1}\right)$ and $w$ is the fuel load $\left(\mathrm{kg} \mathrm{m}^{-2}\right)$. Note the apparent inconsistency in the units Eq. (5), in which $q$ is in $K s^{-1}$, whereas the RHS appears to be $K \mathrm{~ms}^{-1}$, is due to the usual definition of fuel load as mass per area. We assume here that the fuel over the surface area is converted to a volume heat source within the cell, so $w$ is considered to have units of $\mathrm{kg} \mathrm{m}^{-3}$ in the model.

The mass loss rate is fuel dependent but can be approximated as linear for most fuels (Rothermel, 1972, McAllister, 2019). This is a free parameter in the model, and here we pick a fuel load of $25 t h a^{-1}$ with a combustion time of $10 \mathrm{~s}$, giving a mass loss rate of $0.25 \mathrm{~kg} \mathrm{~m}^{-2} \mathrm{~s}^{-1}$. With $H \sim 10^{7} \mathrm{~J} \mathrm{~kg}^{-1}$ and $c_{p} \sim 10^{3} \mathrm{~J} \mathrm{~K}^{-1}$ for typical wildfire fuels this gives an estimated $q \sim 2500 \mathrm{~K} \mathrm{~s}^{-1}$ for the thermal source term. This is a significant simplification of the complexities of the combustion process but serves to provide a heat source of the correct order of magnitude into the simulation (Byram and Fons, 1952).

\subsection{Scenarios}

The two scenarios investigated are shown in Fig. 2, where the shading represents the elevation above ground level. Both scenarios were modelled as isolated ridges with a length of $2 \mathrm{~km}$. The maximum elevation in both scenarios was set to $250 \mathrm{~m}$ giving an uphill slope in both scenarios of approximately $10^{\circ}$ and a downhill slope in scenario $A$ of approximately $20^{\circ}$. Scenario $A$ used a fire line $50 \mathrm{~m}$ from the ridge line on the lee side of the hill and $500 \mathrm{~m}$ in length. Scenario $B$ used a circular fire of radius $100 \mathrm{~m}$ located in a ' $\mathrm{V}$ ' shaped canyon on the upwind side of the hill with a wall angle of $20^{\circ}$. The $10 \mathrm{~m}$ wind speed in both scenarios was set to $10 \mathrm{~m} \mathrm{~s}^{-1}$.

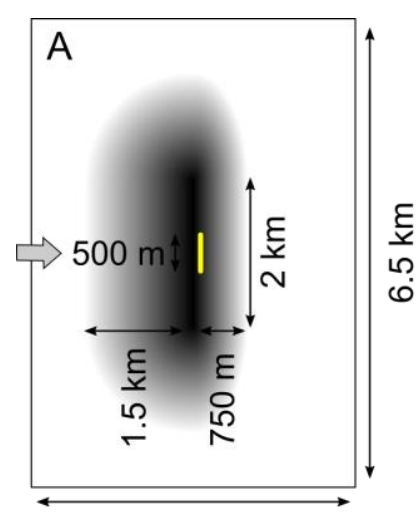

$4.5 \mathrm{~km}$

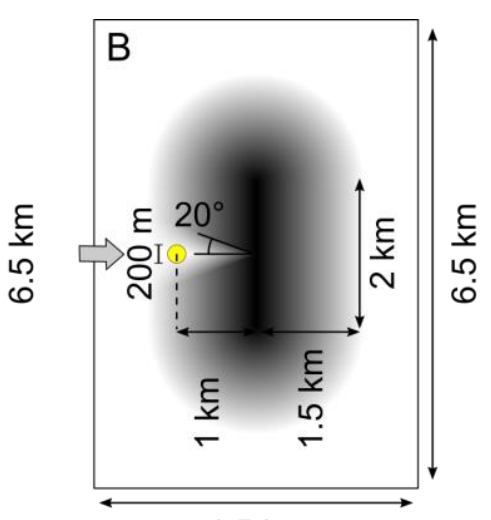

$4.5 \mathrm{~km}$

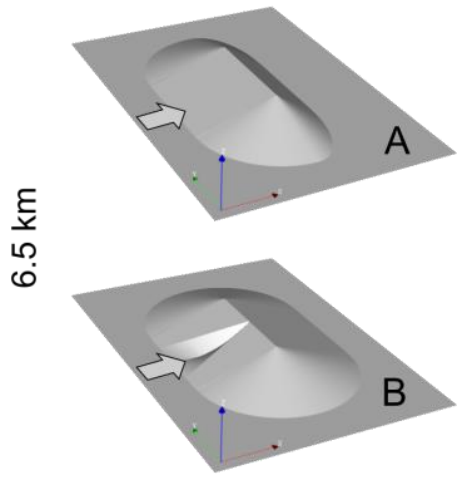

Figure 2. Scenarios used in study. Left: schematic set-up where shading represents the height of the elevation from white $=0 \mathrm{~m}$ to black $=250 \mathrm{~m}$. Yellow areas represent flaming regions used in each scenario. Right: $3 \mathrm{D}$ visualizations of elevation. A: flow over a ridge with fire line on lee slope, B: flow through a ' $V$ ' shaped canyon aligned with the wind.

\section{RESULTS}

Visualisation of the flow and temperature fields as well as the individual firebrands for scenario $A$ are shown in Fig. 3. A similar visualisation of the flow and temperature fields as well as the firebrands is shown in Fig. 4 for scenario $B$. The visualisations show a close-up of the fire from an angle above the ridge looking downwards onto the fire. The fire is shaded using a temperature scale ranging from yellow $\left(1000{ }^{\circ} \mathrm{C}\right)$ through grey to transparent at zero. Velocity vectors are plotted using a hue colour scale ranging from $0 \mathrm{~m} \mathrm{~s}^{-1}$ (blue) to $20 \mathrm{~m} \mathrm{~s}^{-1}$ (red). The firebrands are shown also using a hue colour scale, but shaded by height from 0 $m$ (blue) to $300 m$ 
(red). After an initial transient phase of around $100 \mathrm{~s}$ a complex wake develops with two persistent vortices at the edges of the fire. A density map of the number of firebrands $N$ within each $10 \mathrm{~m}$ computational grid cell at ground level is shown in Fig. 5 , shaded using a logarithmic scale. Despite the complexity of the wake pattern some structure resulting from the edge vortices can be seen in this map.

The density map of firebrands for scenario $B$ is shown in Fig. 5. The dynamics in this scenario are very different to scenario $A$, with a single stable vortex resulting from the wind field interaction with the canyon. This vortex breaks down in the free stream over the ridge dispersing firebrands over a thin strip. This structure appears insensitive to grid resolution and the placement of the fire source within the canyon. Moving the source slightly (on the order of a few grids cells in each direction) from the position shown in Fig. 4 appears to have no effect on the formation of the structure.

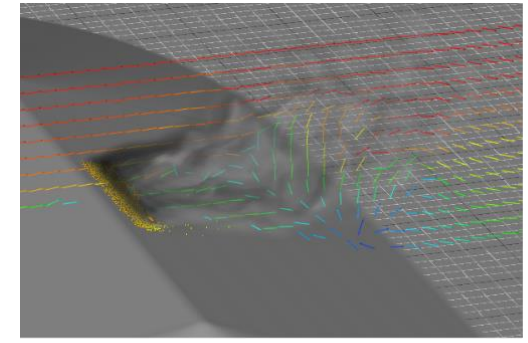

$t=100 s$

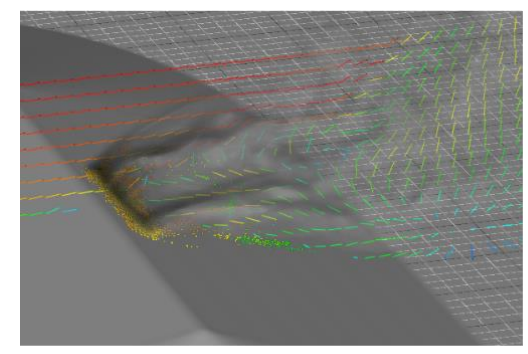

$t=200 s$

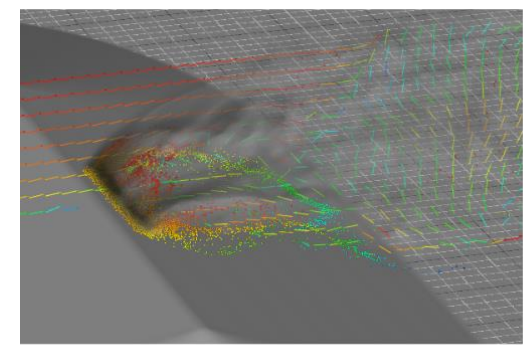

$t=300 s$

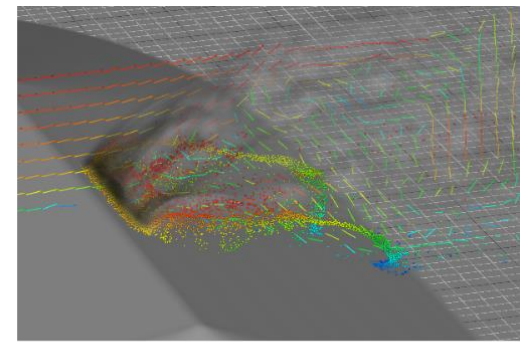

$t=400 s$

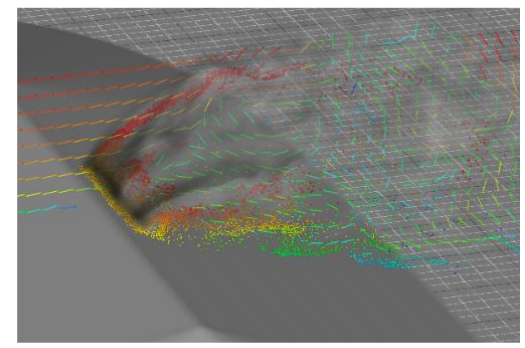

$t=500 s$

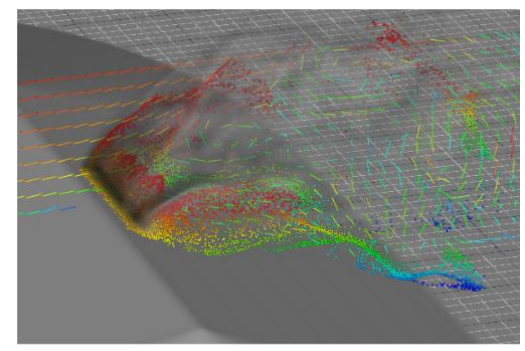

$t=600 s$

Figure 3. Firebrands, flow field and temperature field from scenario A, a lee-side fire perpendicular to a ridge.

\section{DISCUSSION AND CONCLUSION}

Buoyant plumes in an atmospheric boundary layer typically result in a counter-rotating vortex pair (CVP) (Cunningham et al., 2005, Forthofer and Goodrick, 2011, Thurston et al., 2017) with characteristics similar to a jet in a cross-flow (Mahesh, 2013). This appears to be a complex, yet robust, phenomenon resulting from the interaction between a vertical momentum source (the plume) and a horizontal cross-flow field. It appears that the CVP formation is caused by the tilting and folding of the plume shear layer in the cross flow (Morton, 1996, Mahesh, 2013). The CVP phenomenon is a fundamental aspect of the plume structure (Cunningham et al., 2005) and is therefore directly linked to transport of firebrands in wildfires.

The firebrand dynamics in all the simulations within this study appear to result from terrain-dependent effects on the CVP structure. Scenario $A$ involves flow of wind over a ridge. In the absence of a heat source, the steady-state structure is a low-pressure vertically aligned recirculation region on the lee slope of the ridge. The addition of a heat source and subsequent CVP to this flow structure appears to flatten and tilt the CVP around the axis of the incident wind, resulting in elongated recirculation regions at the edges of the fire line. These can be seen in Fig. 3 from $300 \mathrm{~s}$ onwards. Scenario $B$ involves the channelling of flow through a narrowing ' $\mathrm{V}$ ' shaped 
canyon and results in the formation of a single jet-like vortex transporting firebrands upwards and over the top of the canyon. Although more work is required to fully understand the complexities of the flow, a simple explanation for this behaviour may be that the CVP pair is artificially forced together by the terrain resulting in a single vortex. We hypothesise that once a particular rotational sense gains hold, it dominates the vortex motion with a resultant rotation determined by initial fluctuations during formation. At the top of the canyon the jet breaks down in the free stream, dispersing firebrands over a thin area. This dispersal can be seen to be consistent with a single vortex, with a single ' $\mathrm{S}$ ' shaped pattern on the density map.

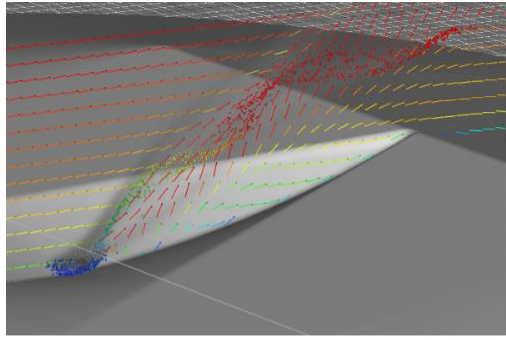

$t=400 s$

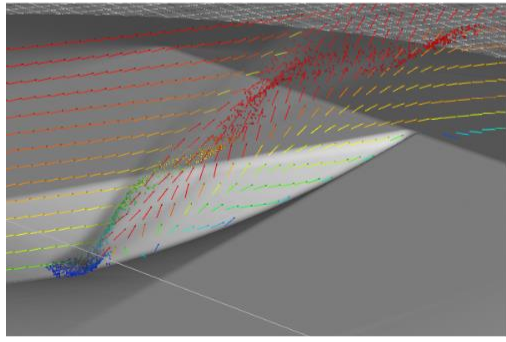

$t=600 s$

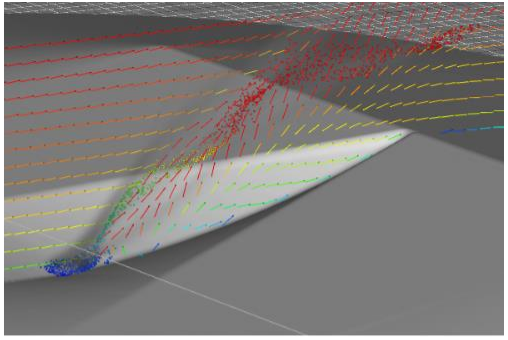

$t=800 s$

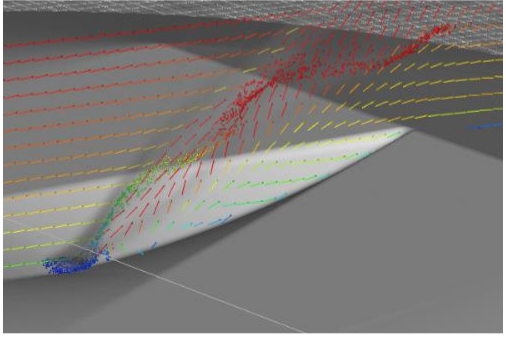

$t=1000 s$

Figure 4. Firebrands, flow field and temperature field from scenario B.
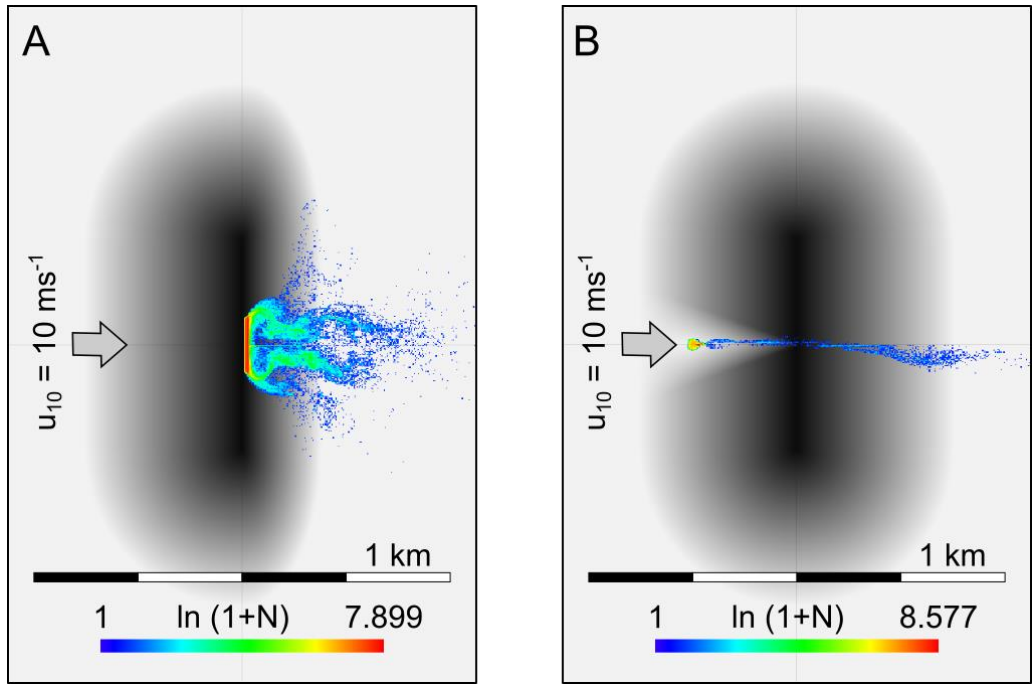

Figure 5. Density map of firebrands for scenario A (left) and scenario B (right)

It is well-known that interaction of wind and fires results in complex flow patterns. The key result of this study is that incorporating terrain adds a further layer of complex flow behaviour that can possibly dominate the resulting fire behaviour. The resulting flow patterns appear, at least in some cases, to be consistent with the modifications to an underlying CVP structure. This almost certainly means that simplified models of firebrand transport, such as a ballistic model along a plume centreline, cannot be used to model firebrand dispersal as the full CVP structure must be taken into account. However, simplified models based on CVP assumptions may be viable in some circumstances when the terrain is simple. For example, ongoing work using this model has shown that vertical ember showers result from the downstream dissipation of the CVP structure holding firebrands in a helical stream. This work will be published in a future study, along with structured validation of all underlying steps involved in the modelling. The work presented here, albeit for highly idealized scenarios, 
Hilton et al., Wind-terrain effects on firebrand dynamics

can give some insight into the complexity of flow structure when terrain is involved and could be used to guide research such as this into improved firebrand transport models for wildfire prediction and risk management.

\section{ACKNOWLEDGMENTS}

This work was funded in part by the Bushfire and natural Hazards Cooperative Research Centre.

\section{REFERENCES}

Blanchi, R., Leonard, J., Leicester, R.H., Bushfire Risk at the Rural/Urban Interface. Australasian Bushfire Conference, 2006.

Byram, G.M., Fons, W.L. (1952). Thermal properties of forest fuels. Interim Technical Report AFSWP - 404. Division of Fire Research, Forest Service, U.S. Department of Agriculture. 34 p.

Chorin, A.J. (1967), The numerical solution of the Navier-Stokes equations for an incompressible fluid, Bull. Am. Math. Soc., 73, 928-931

Cruz, M.G., Sullivan, A.L., Gould, J.S., Sims, N.C. Bannister, A.J., Hollis, J.J., Hurley, R.J. (2012). Anatomy of a catastrophic wildfire: The Black Saturday Kilmore East fire in Victoria, Australia, Forest Ecology and Management 284, 269-285

Cunningham, P., Goodrick, S.L., Hussaini, M.Y., Linn R.R., (2005). Coherent vortical structures in numerical simulations of buoyant plumes from wildland fires. International Journal of Wildland Fire, 14, 61-75.

Ellis, P.F.M. (2013). Firebrand characteristics of the stringy bark of messmate (Eucalyptus obliqua) investigated using non-tethered samples, International Journal of Wildland Fire, 22, 642-651.

Forthofer, J.M., Goodrick, S.L., (2011). Review of vortices in wildland fire. Journal of Combustion 2011, 114.

Hilton, J.E., Cleary P.W. (2012). Raceway formation in laterally gas-driven particle beds, Chemical Engineering Science, 80, 306-316.

Koo, E., Pagni, P. J., Weise, D. R., Woycheese, J. P. (2010). Firebrands and spotting ignition in large-scale fires, International Journal of Wildland Fire, 19, 818-843.

Lahaye, S. Sharples, J., Matthews, S., Heemstra, S., Price, O., Badlan, R., (2018). How do weather and terrain contribute to firefighter entrapments in Australia? International Journal of Wildland Fire 27, 85-98.

Liu, X.-D., Osher, S., Chan, T., (1994). Weighted essentially non-oscillatory schemes, Journal of Computational Physics, 115:200-212.

Mahesh, K. (2013), The Interaction of Jets with Crossflow, Annual Review of Fluid Mechanics, 45, 379-407.

McAllister, S., (2019). The Role of Fuel Bed Geometry and Wind on the Burning Rate of Porous Fuels, Frontiers in Mechanical Engineering, 5, 1-9.

Morton, B.R., Ibbetson, A. (1996). Jets Deflected in a Crossflow, Experimental Thermal and Fluid Science, 12, 112-133.

Nicoud, F., Ducross, F., (1999). Subgrid-scale stress modelling based on the square of the velocity gradient tensor. Flow Turbulence and Combustion, 62, 183-200.

Rothermel, R. C. (1972). A mathematical model for predicting fire spreading wildland fuels. USDA Forest Service Research Paper INT-115.

Raposo, J.R., Cabiddu, S., Viegas, D.X., Salis, M., Sharples, J., (2015). Experimental analysis of fire spread across a two-dimensional ridge under wind conditions, International Journal of Wildland Fire 24, 10081022.

Simpson, C.C., Sharples, J.J., Evans, J.P., (2014). Resolving vorticity-driven lateral fire spread using the WRFFire coupled atmosphere-fire numerical model, Natural Hazards and Earth System Sciences 14 (9), 23592371

Sharples, J.J., McRae, R.H.D., Wilkes, SR., (2012). Wind-terrain effects on the propagation of wildfires in rugged terrain: fire channeling, International Journal of Wildland Fire 21, 282-296.

Spark (2019), http://research.csiro.au/spark/

Thomas, C.M., Sharples, J.J., Evans, J.P. (2019). The terminal velocity assumption in simulations of longrange ember transport. Mathematics and Computers in Simulation. In press.

Thurston, W., Kepert, J. D., Tory, K. J., Fawcett, R. J. B. (2017). The contribution of turbulent plume dynamics to long-range spotting, International Journal of Wildland Fire, 26, 317-330.

Viegas, D.X., Pita, L.P. (2004) Fire spread in canyons. International Journal of Wildland Fire, 13, 253-274.

Wadhwani, R., Sutherland, D., Ooi, A., Moinuddin, K., Thorpe, G. (2017). Verification of a Lagrangian particle model for short-range firebrand transport. Fire Safety Journal. 91, 776-783.

Wadhwani, R., Sutherland, A., Moinuddin, Simulated transport of short-range embers in an idealised bushfire. Proceedings for the 6th International Fire Behavior and Fuels Conference, Sydney, Australia.

Wesseling, P. (1992). An introduction to multigrid methods. Chichester; New York, Wiley. 